\title{
An amino-bisphosphonate targets MMP-9- expressing macrophages and angiogenesis to impair cervical carcinogenesis
}

\author{
Enrico Giraudo, Masahiro Inoue, and Douglas Hanahan \\ Department of Biochemistry and Biophysics, Diabetes Center, and Comprehensive Cancer Center, University of California, San Francisco, \\ San Francisco, California, USA.
}

\begin{abstract}
A mouse model involving the human papillomavirus type-16 oncogenes develops cervical cancers by lesional stages analogous to those in humans. In this study the angiogenic phenotype was characterized, revealing intense angiogenesis in high-grade cervical intraepithelial neoplasias (CIN-3) and carcinomas. MMP-9, a proangiogenic protease implicated in mobilization of VEGF, appeared in the stroma concomitant with the angiogenic switch, expressed by infiltrating macrophages, similar to what has been observed in humans. Preclinical trials sought to target MMP-9 and angiogenesis with a prototypical MMP inhibitor and with a bisphosphonate, zoledronic acid (ZA), revealing both to be antiangiogenic, producing effects comparable to a $M m p 9$ gene KO in impairing angiogenic switching, progression of premalignant lesions, and tumor growth. ZA therapy increased neoplastic epithelial and endothelial cell apoptosis without affecting hyperproliferation, indicating that ZA was not antimitotic. The analyses implicated cellular and molecular targets of ZA's actions: ZA suppressed MMP-9 expression by infiltrating macrophages and inhibited metalloprotease activity, reducing association of VEGF with its receptor on angiogenic endothelial cells. Given its track record in clinical use with limited toxicity, ZA holds promise as an "unconventional" MMP-9 inhibitor for antiangiogenic therapy of cervical cancer and potentially for additional cancers and other diseases where MMP-9 expression by infiltrating macrophages is evident.
\end{abstract}

\section{Introduction}

Cervical cancer is the second most common malignant disease among women worldwide (1), and the "high-risk" human papillomavirus (HPV) types, such as HPV-16 and HPV-18, are found in $80-90 \%$ of invasive cervical cancers as well as in distinctive premalignant stages (2) of cervical intraepithelial neoplasia (CIN). Angiogenesis has been clinically associated with the progression of CIN lesions to invasive cervical carcinoma; neovascularization develops at the stromal/epithelial junction of CIN lesions, characterized by marked increases in vessel density and size $(3,4)$. This progressively intensifying angiogenesis is associated with upregulated expression of VEGF (4-6) and extracellular proteases, including MMP-9 $(7,8)$.

In this report, we have used a mouse model of human cervical carcinogenesis (estrogen-treated K14-HPV16 female transgenic mouse, or $\mathrm{HPV} / \mathrm{E}_{2}$ mouse) to study the mechanisms regulating angiogenesis and malignant progression and to begin exploring possible antiangiogenic therapies with the potential to prevent and/or regress cervical carcinoma. K14-HPV16 mice express the HPV-16 early genes under the control of the keratin 14 promoter, resulting in targeted oncogene expression in keratin 14-positive squamous epithelial cells, a natural host cell target for HPV infec-

Nonstandard abbreviations used: $\mathrm{BP}$, bisphosphonate; $\mathrm{CEP}$, circulating endothelial progenitor cell; CIN, cervical intraepithelial neoplasia; $\mathrm{E}_{2}, 17 \beta$-estradiol; EC,

endothelial cell; HPV, human papillomavirus; $\mathrm{HPV} / \mathrm{E}_{2}$, estrogen-treated K14-HPV16 female transgenic; HSC, hematopoietic stem cell; IHC, immunohistochemistry; MMPI, MMP inhibitor; N-BP, nitrogen-containing (amino-) bisphosphonate; $\mathrm{N} / \mathrm{E}_{2}$, nontransgenic estrogen-treated female; PFA, paraformaldehyde; 1,10 Phe, 1,10 phenanthroline; PT, prevention trial; RFU, relative fluorescence units; RT, regression trial; SCC, squamous cell carcinoma; $T_{\mathrm{a}}$, annealing temperature; $Z A$, zoledronic acid. Conflict of interest: The authors have declared that no conflict of interest exists.

Citation for this article: J. Clin. Invest. 114:623-633 (2004)

doi:10.1172/JCI200422087. tion in humans. K14-HPV16 mice develop squamous cell carcinoma (SCC) of the skin with $50 \%$ penetrance over a 12 -month time course in the $\mathrm{FVB} / \mathrm{n}$ mouse strain $(9,10)$. Additionally, if young (4- to 5-week-old) K14-HPV16 females are dosed chronically with time release, low-dose estrogen (17 $\beta$-estradiol; $\left.\mathrm{E}_{2}\right)$, a synchronous progression leading to cervical cancer ensues (11). The HPV $/ \mathrm{E}_{2}$ females develop CIN lesions that progress to invasive SCCs (specifically, cervical carcinomas) at the transformation zone, the site implicated in the genesis of human cervical cancer $(4,12)$; cervical carcinomas begin to appear at 4 months, and by 7 months, $90 \%$ of the mice have invasive cancer. Herein we characterize the angiogenic phenotype in this mouse model of human cervical carcinogenesis and evaluate antiangiogenic therapy with a bisphosphonate (BP) previously approved for use in treating bone metastases to determine its possible use for prevention and therapy of cervical cancer.

\section{Results}

Cervical carcinogenesis and the angiogenic phenotype in $\mathrm{HPV} / \mathrm{E}_{2}$ mice. We evaluated angiogenesis in the distinctive neoplastic stages that arise in the HPV/ $\mathrm{E}_{2}$ transgenic mouse model of cervical cancer. The histopathological progression to cervical malignancy through lowgrade dysplasia (CIN-1/2), to high-grade dysplasia (CIN-3), and then to cervical carcinoma (SCC), is shown in Figure 1A, in comparison with nontransgenic estrogen-treated female $\left(\mathrm{N} / \mathrm{E}_{2}\right)$ control mice; at the defined end point of 7 months of age, when approximately $90 \%$ of the $\mathrm{HPV} / \mathrm{E}_{2}$ females have invasive cancer, there are no macroscopic LN or distant metastases (not shown). The cervical vasculature was visualized in these lesional stages by CD-31 immunostaining (Figure 1B), revealing modest neovascularization below the stromal/epithelial junction in $\mathrm{CIN}-1 / 2$ lesions, followed by intense angiogenesis in CIN-3 lesions and cervical carcinomas, 

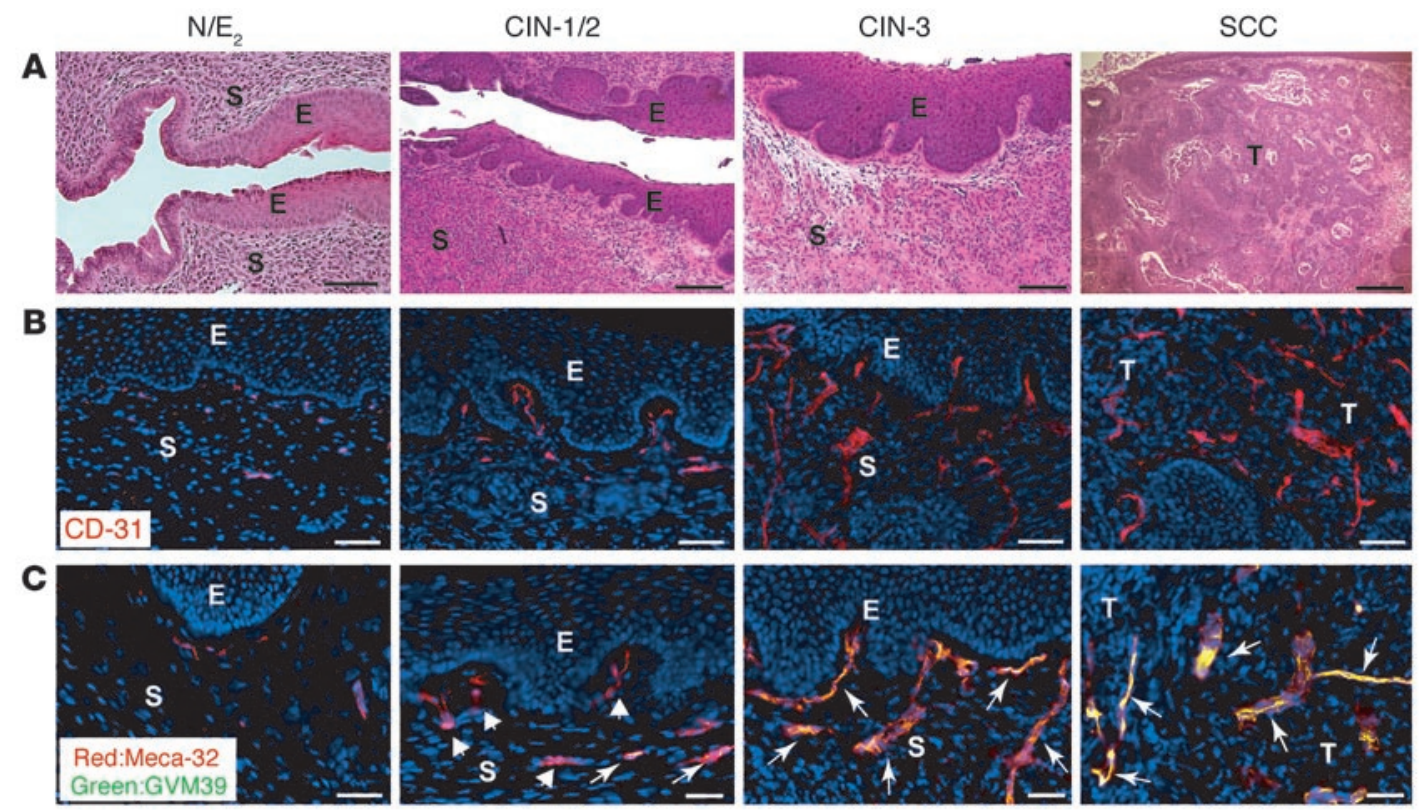

\section{Figure 1}

Stages of cervical carcinogenesis and angiogenic profile of HPV/E 2 mice. (A) H\&E staining shows the steps of tumor progression. (B) Blood vessels (red) were detected with anti-CD-31 Ab; nuclei were visualized by DAPI (blue). The number of vessels per field were: $N / E_{2}, 8.2 \pm 1.2 ; C I N-1 / 2$, $14.8 \pm 1.1$; CIN-3, $32.2 \pm 1.7$; SCC, $35.2 \pm 1.8$. Significant differences were observed between CIN-3/SCC and CIN-1/2 $(P<0.01)$, CIN-3/SCC and $\mathrm{N} / \mathrm{E}_{2}(P<0.01)$, and $\mathrm{CIN}-1 / 2$ and N/E $2(P<0.01)$, but not between $\mathrm{CIN}-3$ and SCC $(P=0.309)$. (C) VEGF-A/VEGF-R2 complex was detected with GVM39 Ab (green) and ECs with anti-Meca-32 Ab (red). VEGF/VEGF-R2 complex colocalizes with most vessels in CIN-3 and SCC and with a subset in CIN-1/2 (arrows). Arrowheads in CIN-1/2 indicate vessels that do not bind GVM39 Ab. Minimal GVM39 staining was detected in $\mathrm{N} / \mathrm{E}_{2}$ stroma. The percentage of VEGF-A/VEGF-R2-labeled vessels was quantitated: $\mathrm{N} / \mathrm{E}_{2}, 8.6 \pm 1.2 ; \mathrm{CIN}-1 / 2,44.1 \pm 2.9 ; \mathrm{CIN}-3,90.2 \pm 3.1 ; \mathrm{SCC}$, $91.4 \pm$ 3.4. Significant differences were observed between $\mathrm{CIN}-3 / \mathrm{SCC}$ and $\mathrm{CIN}-1 / 2(P<0.01), \mathrm{CIN}-3 / \mathrm{SCC}$ and N/E $\mathrm{E}_{2}(P<0.01)$, and $\mathrm{CIN}-1 / 2$ and $\mathrm{N} / \mathrm{E}_{2}(P<0.01)$. CIN-3 and SCC were not significantly different $(P=0.690)$. Values are mean $\pm \mathrm{SEM}$. $P$ values were calculated using the Wilcoxon test. Scale bars in A and C: $50 \mu \mathrm{m}\left(\mathrm{N} / \mathrm{E}_{2}\right) ; 25 \mu \mathrm{m}(\mathrm{CIN}-1 / 2, \mathrm{CIN}-3$, and SCC). Scale bars in B: $50 \mu \mathrm{m}$. E, normal or dysplastic cervical epithelia; $\mathrm{S}$, stroma; T, tumor cells.

as previously observed in human cervical carcinogenesis (3). Given that VEGF-A and its principal signaling receptor, VEGF-R2/flk-1, are commonly involved in angiogenic switching and tumor growth $(13,14)$ and are implicated in human cervical neoplasia, we assessed their possible involvement in the angiogenic phenotype. Using double-labeled immunofluorescence with a $\mathrm{mAb}$ that specifically recognizes VEGF in complex with VEGF-R2 (GVM39) (15), along with the Meca-32 Ab to visualize endothelial cells (ECs), we observed a modest increase in the VEGF/VEGF-R2 complex in vessels below the stromal/epithelial junction of $\mathrm{CIN}-1 / 2$ lesions and a substantial increase in the high-grade CIN-3 lesions and invasive cervical tumors as compared with the normal cervix (not shown) and $\mathrm{N} / \mathrm{E}_{2}$ mice (Figure $1 \mathrm{C}$ ).

$M M P-9$ and macrophages are associated with the angiogenic vasculature in dysplasias and tumors. Motivated by previous studies in mouse models functionally implicating MMP-9/gelatinase B as a regulator of VEGF signaling and angiogenesis in other organs (13, $16-19)$, as well as in broader cancer phenotypes $(20,21)$, and by reports that MMP-9 is upregulated in human cervical neoplasias $(7,8)$, we evaluated expression of MMP-9 and related MMPs during cervical carcinogenesis. RT-PCR analysis of nine different MMPs revealed that only MMP-9 was markedly upregulated during tumor progression in the cervix of $\mathrm{HPV} / \mathrm{E}_{2}$ mice (Figure 2A). By contrast, a related protease, MMP-2, was constitutively expressed in all stages (Figure 2A); mRNAs for seven other MMPs were analyzed, and we observed unchanged or undetectable levels of expression (see Supplemental Figure 1, available at http://www. jci.org/cgi/content/full/114/5/623/DC1).

Using immunohistochemistry (IHC), we confirmed the RNA analysis, detecting MMP-9 expression in scattered cells, both in the stroma adjacent to the epithelial interface of CIN lesions and in tumors (Figure 2B); no such cells were detected in cervix of control or $\mathrm{N} / \mathrm{E}_{2}$ mice (Figure 2B, and data not shown). To ascertain whether the appearance of MMP-9 RNA and protein immunoreactivity reflected proteolytically active enzyme, we performed gelatin zymography, which detects both latent pro- and active forms of the enzyme. We observed, consistent with RNA expression, an increase in both forms of MMP-9, particularly in the high-grade dysplasias (CIN-3) and carcinomas, compared with controls (Figure 2C). In contrast, we detected only a slight increase in abundance of the MMP-2 pro form and no evident upregulation of active MMP-2 (Figure 2C). We have substantiated and further quantified the profile of protease activity in these stages using a biochemical assay for gelatinase activity, which showed statistically significant upregulation of total gelatinolytic activity in CIN-3 lesions and SCC as compared with controls (Figure 2D). Consistent with the zymogram analysis (Figure 2C), no significant difference in activity was observed between CIN-3 and SCC (Figure 2D).

It has previously been demonstrated in the related $\mathrm{K} 14$ HPV16 skin cancer model, that MMP-9 is supplied by bone marrow-derived cells rather than by transformed epithelial cells (17). To identify the MMP-9-expressing cells in the cervix, 
A

Stages $N / E_{2}$ CIN-1 CIN-2 CIN-3 SCC

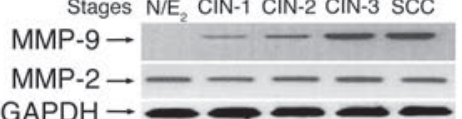

$\mathrm{GAPDH} \rightarrow \longrightarrow$

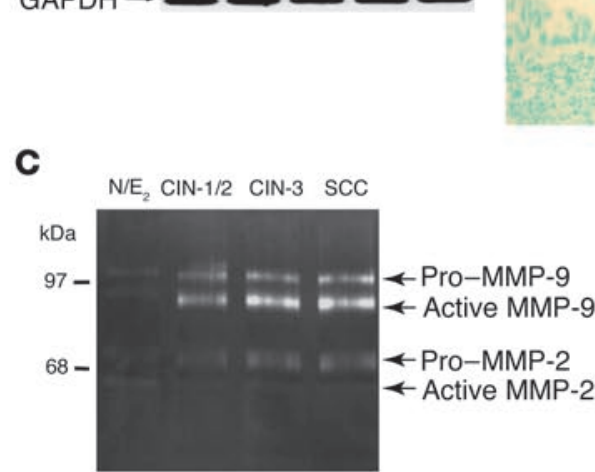

E

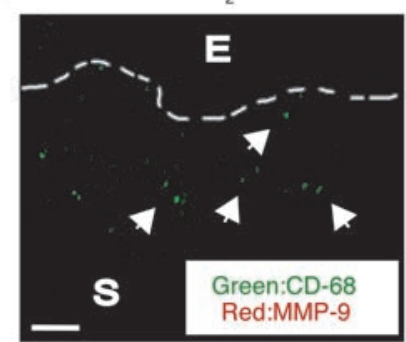

B $\quad \mathrm{N} / \mathrm{E}_{2}$

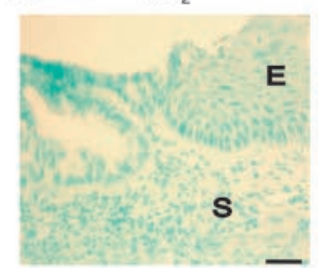

$\mathrm{CIN}-1 / 2$
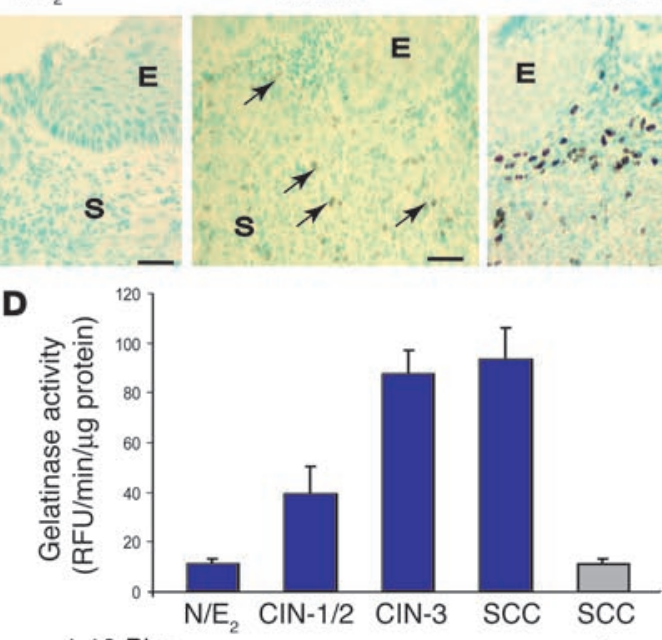

1,10 Phe -
CIN-3

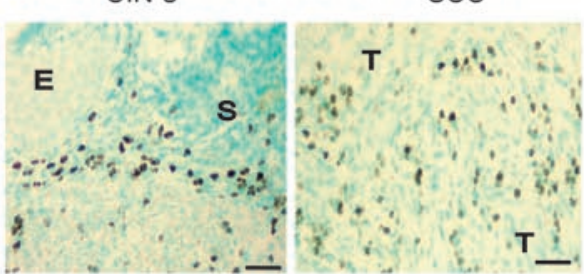

$\mathrm{CIN}-1 / 2$

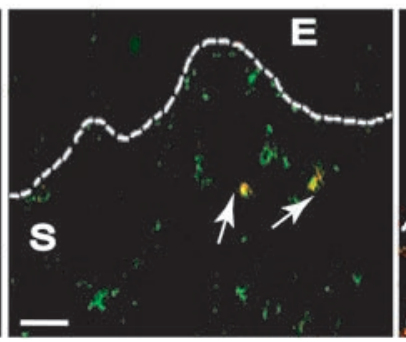

$\mathrm{CIN}-3$
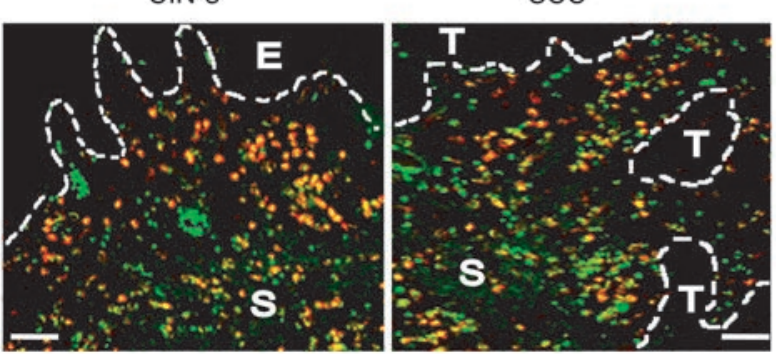

Figure 2

MMP-9 expression and activity is upregulated in macrophages during tumor progression. (A) RT-PCR analysis revealed increased MMP-9 expression in CIN-3 and SCC as compared with CIN-1/2. No MMP-9 expression was detected in normal cervix (not shown) or in N/E 2 . MMP-2 was equally expressed at all stages. (B) Immunohistochemical analysis using an anti-MMP-9 Ab revealed no MMP-9 in N/E 2 cervix and minimal expression in CIN-1/2 lesions (arrows); in contrast, MMP-9 was detected in the stroma proximal to CIN-3 lesions and tumors. (C) Zymography showing gelatinase activity in tissue lysates of different stages. Both pro-MMP-9 (inactive form, $105 \mathrm{kDa}$ ) and active MMP-9 (95 kDa) were upregulated in CIN lesions and tumors as compared with controls. pro-MMP-2 (72 kDa) was slightly increased during progression, but no active MMP-2 (62 kDa) was detected. (D) Gelatinase activity was measured using a fluorescin-gelatin assay in the absence or presence of the MMP inhibitor 1,10 Phe (4 mM). Statistically significant increases in gelatinase activity were observed in CIN lesions and tumors compared with controls: CIN-3/SCC versus CIN-1/2 $(P<0.01)$; CIN-3/SCC versus N/E $2(P<0.01)$; CIN-1/2 versus N/E $2(P<0.01)$; CIN-3 and SCC were not significantly different $(P=0.102)$. Values are mean \pm SEM. $P$ values were calculated using the Wilcoxon test. (E) Colocalization of MMP-9 (red) and macrophages (CD-68, green) was observed in the stroma underlying CIN-3 and surrounding SCC. Few MMP-9-expressing macrophages were detected in stroma adjacent to $\mathrm{CIN}-1 / 2$ lesions (arrows). No MMP-9 expression was observed in macrophages in N/E $\mathrm{E}_{2}$ mice (arrowheads). Scale bars: $50 \mu \mathrm{m}\left(\mathrm{N} / \mathrm{E}_{2}\right) ; 25 \mu \mathrm{m}(\mathrm{CIN}-1 / 2, \mathrm{CIN}-3$, and $\mathrm{SCC})$.

we performed immunostaining for markers of various inflammatory cell types, comparing the different stages of tumor progression in $\mathrm{HPV} / \mathrm{E}_{2}$ mice. We observed a substantive increase in the number of infiltrating macrophages, as assessed by tissue immunostaining with Ab's to F4/80 (data not shown) and CD68 (Supplemental Figure 2); by contrast, the abundance of other leucocytes was not appreciably altered (Supplemental Figure 2). Using double-labeled immunofluorescence, we detected MMP-9 expression in macrophages infiltrating the stroma underlying CIN-3 lesions and in tumors (Figure 2E). Notably, the increase in MMP-9-expressing macrophages was concordant with the intense angiogenesis seen in CIN-3 as compared with CIN-1/2 (Figure 2E). Only slight and sporadic MMP-9 immunoreactivity was detected in ECs of the angiogenic vasculature or in stromal fibroblasts associated with the tumors (data not shown). Thus, these particular infiltrating macrophages seem unable to induce
MMP-9 in other stromal cell types, but rather themselves serve as the major source of MMP-9.

The nitrogen-containing (amino-) bisphosphonate zoledronic acid impairs angiogenesis, progression, and growth of cervical carcinomas. Molecular and histological evaluation of cervical cancer progression in humans and in $\mathrm{HPV} / \mathrm{E}_{2}$ mice presents striking parallels between the two pathways $(5,6,11,12)$. The HPV/ $\mathrm{E}_{2}$ mouse model thus presents a platform for preclinical testing of drugs designed to interrupt critical pathways implicated in angiogenesis and lesional progression of cervical carcinoma. Given the above data implicating MMP-9 in angiogenesis and progression, we became interested in assessing the effects of MMP inhibitors (MMPIs) on tumor growth and angiogenesis. Therapeutic trials in a transgenic mouse model of neuroendocrine cancer had previously demonstrated the capability of a prototypical MMPI, BB-94, to inhibit angiogenesis and tumor growth when treatment was initiated during premalignant stages 

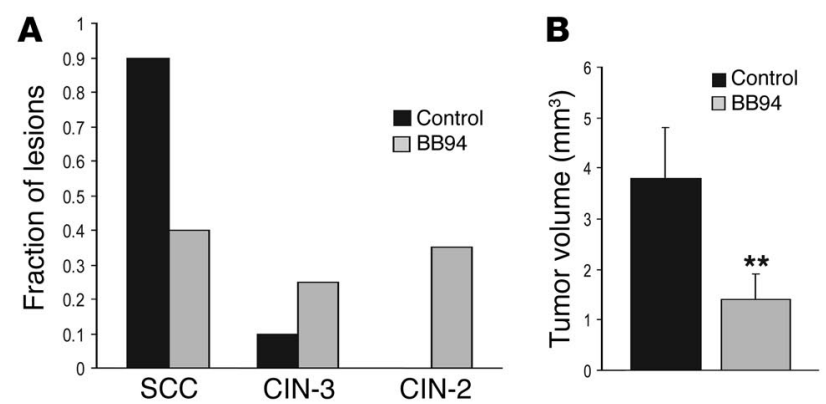

$(13,22)$. Therefore, we conducted analogous early-stage trials targeting $\mathrm{CIN}-2 / 3$ lesions with BB94, which produced reductions in the density of the angiogenic neovasculature (data not shown) and in tumor incidence and tumor growth/burden (Figure 3, A and B).

While encouraged, we were aware that human clinical trials in various disease indications using BB94 and other active site-targeted MMPI had failed due to side effects and toxicity as well as poor efficacy against end-stage tumors (23). We were thus led to consider a much different agent, zoledronic acid (ZA; Zometa), a nitrogen-containing (amino-) bisphosphonate (N-BP) that is FDA approved to reduce skeletal complications of bone metastasis in patients with multiple myeloma and several solid tumor types with minimal side effects $(24,25)$. Recent studies in cell culture and xenotransplant tumor models have variously suggested that ZA and other BPs have antiangiogenic and MMP-inhibitory activity (26-31). These data led us to test ZA on cervical neoplasias in $\mathrm{HPV} / \mathrm{E}_{2}$ mice.

We treated female mice bearing CIN-3 lesions with ZA $(100 \mu \mathrm{g} / \mathrm{kg}$ daily, subcutaneously) or with vehicle alone for 6 weeks, asking whether ZA could prevent tumor formation and/or affect angiogenesis (in a prevention trial [PT]). Although ZA is inoculated intravenously on a monthly regimen in humans, a number of preclinical studies suggested that it was best supplied in a daily regimen in mice, reflecting much different pharmacokinetics $(26,27)$. Fluorescin-lectin perfusion and immunostaining with Meca-32 Ab revealed a decrease of $56 \%$ in the number of blood vessels proximal to the basement membrane in dysplastic lesions and cervical carcinomas from ZA-treated mice compared with analogous lesions in controls (Figure 4, A and B). At the defined end point (5 months of age), the control mice had a tumor incidence of $85 \%$ (Figure 4C). Remarkably, ZA was able to limit the tumor incidence to $30 \%$ (Figure 4C) and reduce tumor volume $61 \%$ (Figure $4 \mathrm{D}$ ).

Next, to assess the effects of ZA on the growth of established cervical carcinomas, we performed a regression trial (RT), treating the mice with ZA for 1 month starting at 5 months of age, when $85 \%$ had malignant tumors and the remainder had CIN-3 lesions. One month later the control mice had a
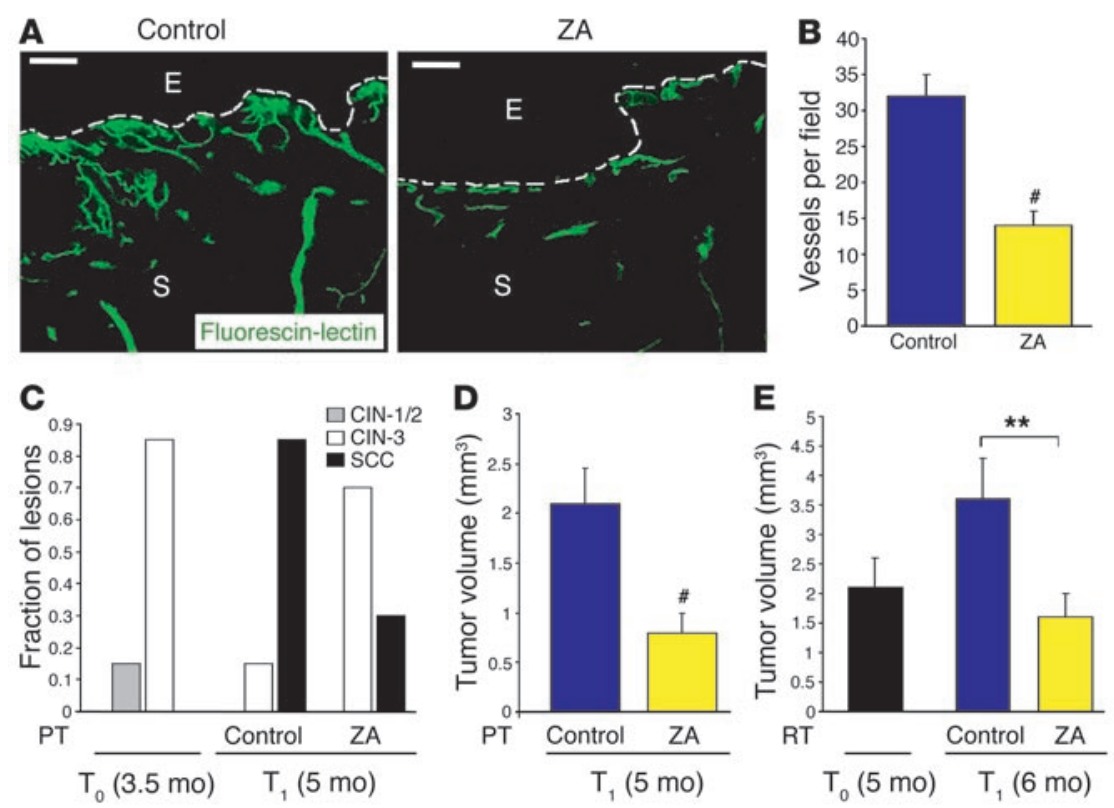

Figure 4

$\mathrm{ZA}$ inhibits angiogenesis and reduces tumor incidence and growth. (A) Perfusion of HPV/E 2 control mice and ZA-treated mice ( 6 weeks of treatment; PT) with fluorescin-lectin revealed dramatic changes in the 3-dimensional organization of the vasculature proximal to CIN-3 lesions of treated mice at 5 months of age. (B) Vessels' density, as assessed by Meca-32 immunostaining, was significantly reduced in ZA-treated mice as compared with controls ( $56 \%$ reduction). Results are mean \pm SEM of five fields per mouse from a total of eight mice. (C) $\mathrm{CIN}-2 / 3$ lesion-bearing mice ( $T_{0}$, beginning of treatment; 3.5 months old) treated with ZA or vehicle for 6 weeks (PT) showed a $55 \%$ reduction in the tumor incidence at the end of the treatment $\left(T_{1} ; 5\right.$ months old $)$ ( $n=16$ control, $n=10$ ZA-treated). (D) Tumor volume of ZA-treated mice was reduced by $61 \%$ compared with untreated controls (PT; $n=16$ control, $n=10$ ZA-treated). (E) Mice bearing SCC ( $T_{0} ; 5$ months old) treated with ZA for 1 month $\left(T_{1}\right)$ in a RT showed a $57 \%$ decrease in tumor volume ( $n=15$ control, $n=10$ ZA-treated). Values are mean \pm SEM. ${ }^{* \star} P<0.01 ;{ }^{\#} P<0.001$. $P$ values were calculated using the Wilcoxon test. Tumor volume and histological scores were determined as described in Methods. Scale bar: $50 \mu \mathrm{m}$. 


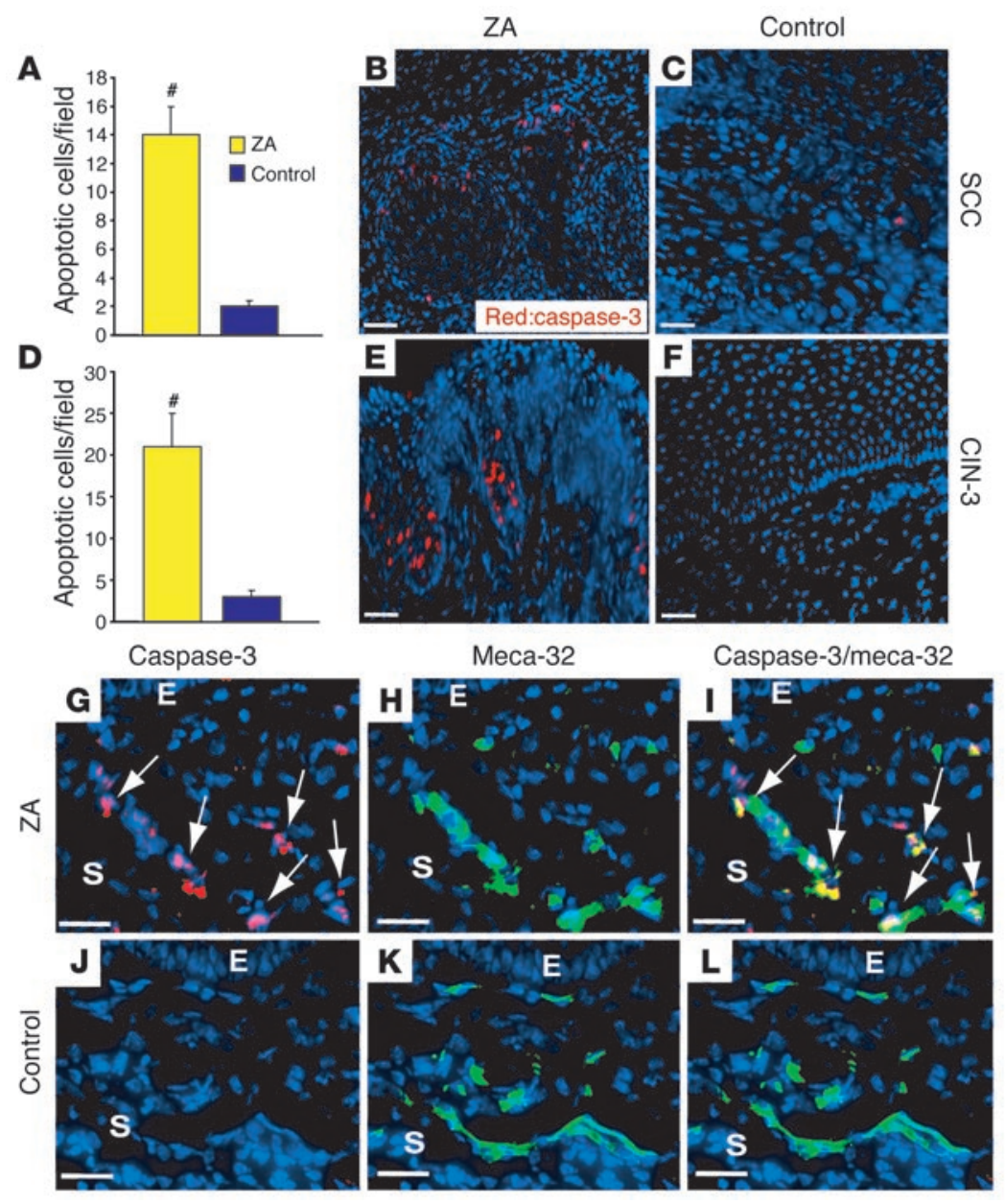

Figure 5

ZA induces apoptosis in epithelial cells and ECs in tumors and $\mathrm{CIN}-3$ lesions. (A-C) A significant increase in apoptosis in cervical carcinomas was observed in ZA-treated mice compared with controls (A) after 6 weeks of treatment (PT) as revealed by caspase- 3 immunostaining ( $P<0.001$, Wilcoxon test). Representative sections of tumors from treated and untreated mice are shown in $\mathbf{B}$ and $\mathbf{C}$. (D-F) Similar increase in apoptosis was detected in CIN-3 epithelium of ZA-treated mice as compared with controls (D); treated versus untreated lesions are exemplified in $\mathbf{E}$ and $\mathbf{F}$. Five fields per mouse were counted to assess the caspase3 -positive cells. Values are mean \pm SEM. Increased apoptosis was observed in vessels in CIN-3 lesions of ZA-treated mice $(\mathbf{G}-\mathbf{I})$ compared with controls $(\mathbf{J}-\mathbf{L})$ as detected by colocalization of Meca-32 (green) with caspase-3 (red). Arrows indicate apoptotic ECs. Scale bars: $50 \mu \mathrm{m}$ (B and $\mathbf{E}) ; 25 \mu \mathrm{m}(\mathbf{C}, \mathbf{F}$, and $\mathbf{G}-\mathbf{I})$.

antimitotic drug, since it does not reduce proliferation of the transformed cervical epithelial cells.

$Z A$ inhibits MMP-9 expression by macrophages and activity in CIN-3 and cervical carcinomas. The ZA trial design was motivated by the above data showing that macrophages infiltrating the stroma of CIN-3 lesions and tumors produced MMP-9, a proangiogenic protease, and by the emerging appreciation of ZA as an inhibitor of angiogenesis and of MMP activity. Moreover, N-BPs have been shown to impair the function of macrophages in vitro $(32,33)$. We asked, therefore, whether ZA therapy was affecting the abundance of infiltrating macrophages, their expression of MMP-9, or the conversion of

of the apoptotic program, as a biomarker for apoptosis, monitoring the presence of its activated form by IHC in ZA-treated mice; we observed a statistically significant increase in cells expressing active caspase- 3 in treated versus control tumors (Figure 5, A-C). ZA treatment also increased the incidence of apoptosis in CIN-3 lesions, whereas fewer apoptotic cells were detected in CIN-3 of control or vehicle-treated mice (Figure 5, D-F). Similar results were obtained using the TUNEL assay to detect DNA fragmentation (data not shown). We further investigated the apoptotic rate in ECs in neoplastic lesions of mice treated with ZA. Double-label immunofluorescence using Meca-32 and caspase-3 Ab's showed an increase in the number of apoptotic ECs within vessels ( $6 \pm 1$ / field, $P<0.001$ ) of the stroma proximal to CIN-3 lesions (Figure $5, \mathrm{G}-\mathrm{I})$ and in cervical carcinomas $(5 \pm 1$ /field, $P<0.001$; data not shown). No apoptotic ECs were detected in analogous lesions from vehicle-treated control mice (Figure 5, J-L).

The squamous epithelium of the cervix in $\mathrm{HPV} / \mathrm{E}_{2}$ mice shows a progressive increase in proliferation rates in the successive stages of carcinogenesis $(11,12)$, motivating us to ask whether ZA was also impacting the cell division cycle. We did not detect significant differences in the rates of proliferation of neoplastic cervical epithelial cells in CIN-3 lesions or cervical carcinomas nor in their adjacent stroma, as compared with untreated controls (see Supplemental Figure 3). Taken together, these data suggest that the biological effects of ZA result from increased apoptosis in epithelial and endothelial cells of the lesions. By contrast, ZA does not appear to act as an
pro-MMP-9 into active MMP-9. We assessed the abundance of both $\mathrm{F} 4 / 80^{+}$(data not shown) and $\mathrm{CD}^{+} 8^{+}$macrophages (Figure 6 ) and of MMP-9-expressing cells in the stroma of the ZA-treated cervix, compared with vehicle controls following a 6-week PT. Using double-labeled immunofluorescence, we detected only a slight decrease in the number of infiltrating macrophages in ZA-treated mice ( $10 \%$ reduction, data not shown). In contrast, we observed a $71 \%$ reduction of MMP- 9 expression in macrophages in the stroma proximal to $\mathrm{CIN}-3$ lesions (Figure $6, \mathrm{~A}$ and $\mathrm{B}$ ) and a $73 \%$ reduction of MMP-9 in macrophages associated with tumors relative to vehicle-treated controls (data not shown). We detected a similar inhibition of MMP-9 expression by macrophages (68\% reduction) in the RT targeting preexisting cervical carcinomas (Figure $6 \mathrm{~B})$. Since ZA did not completely inhibit MMP-9 expression, we measured the total gelatinolytic activity in tissues of ZA-treated compared with control mice by gelatinase assay. Consistent with the immunofluorescence results (Figure 6, A and B), we observed a strong inhibition of the total gelatinolytic activity in ZA-treated mice in both PTs and RTs compared with controls (Figure 6C). We also performed gelatin zymography to reveal the pro form and active forms of MMP-9. In agreement with gelatinolytic biochemical assay, after 6 weeks of treatment (in a PT), ZA reduced the abundance of the MMP-9 pro form $70 \%$ (reflecting the reduced number of MMP-9-expressing macrophages) and almost completely inhibited the activation of latent pro-MMP-9, in that the smaller active form of the protease was barely detectable (Figure 
A
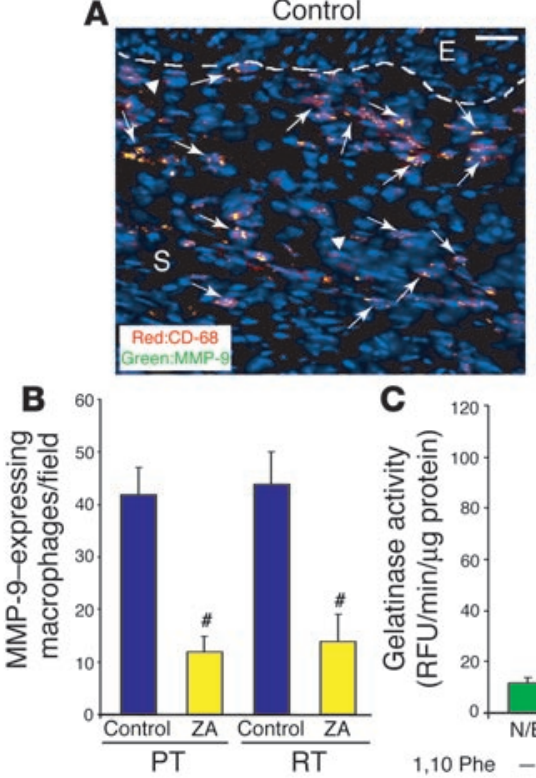

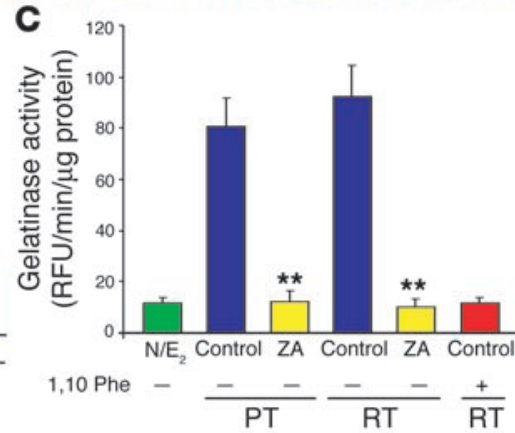

Figure 6

ZA inhibits MMP-9 expression and activation in macrophages. (A) Reduced MMP-9 expression was detected in macrophages in the stroma adjacent to $\mathrm{CIN}-3$ in ZA-treated mice compared with controls as revealed by colocalization of MMP-9 (green) and CD-68 (red). Arrows show MMP-9-expressing macrophages; arrowheads indicate macrophages that do not express MMP-9. (B) Quantification of double-labeled MMP- $9^{+} / \mathrm{CD}-68^{+}$cells (5 fields per mouse) revealed a $71 \%$ reduction in the PT $(n=8$ control, $n=6$ ZA-treated) and a $68 \%$ reduction in the RT ( $n=10$ control, $n=8$ ZA-treated). Similar results were obtained with double-labeled MMP- $9^{+} / \mathrm{F} 4 / 80^{+}$cells (not shown). ${ }^{\sharp} P<0.001$. (C) Gelatinase activity in tissue extracts was measured by incubation with fluorescin-conjugated gelatin in the absence or presence of the MMP inhibitor 1,10 Phe $(4 \mathrm{mM})$. Gelatinase activity was lower in ZA-treated compared with control cervixes in both $\mathrm{PT}$ and $\mathrm{RT}$. ${ }^{* \star} P<0.01$ versus control. N/E $\mathrm{E}_{2}$ indicates estrogen-treated normal cervix. (D) Zymography showing the pro- and active forms of gelatinases in tissue lysates from both control and ZA-treated mice. Pro- and active forms of MMP-9 and MMP-2 are indicated by arrows. Scale bar: $25 \mu \mathrm{m}$. Values are mean \pm SEM. $P$ values were calculated using the Wilcoxon test.

6D). We observed a slight decrease in the abundance of the MMP-2 pro form, although the significance is unclear, since we did not detect appreciable MMP-2 activity in either the untreated control or the ZA-treated neoplastic cervix (Figure 6D). These data suggest a dual effect of ZA: it suppressed the expression of MMP-9 by infiltrating macrophages, and it inhibited the proteolytic activity of the remaining (perhaps preexisting) MMP-9 in the stroma associated with CIN-3 lesions and cervical carcinomas.

Gene KO mice reveal the functional importance of $M M P-9$ for cervical carcinogenesis. The dual MMP-9 inhibitory effects of ZA treatment clearly suggested that MMP-9 expressed by macrophages is critical for tumor angiogenesis and cancer progression in the cervix. Most MMP inhibitors, while possibly selective for a particular protease, typically inhibit multiple MMPs $(23,30,31)$. To assess whether MMP-9 was functionally involved in regulation of angiogenesis and tumor growth, we used a genetic approach involving HPV16 mice carrying gene KOs of either MMP-2 or MMP-9, afforded by the fact that both protease $\mathrm{KO}$ mice are viable $(34,35)$. To functionally evaluate the roles of MMP-9 and MMP- 2 in cervical cancer progression, we analyzed the cervical cancer phenotype in $\mathrm{HPV} / \mathrm{E}_{2}$ mice that had been intercrossed to MMP-9-null or MMP-2-null mice (all maintained in the $\mathrm{FVB} / \mathrm{n}$ background) to render them deficient in one or the other protease. The tumor phenotype was evaluated
D

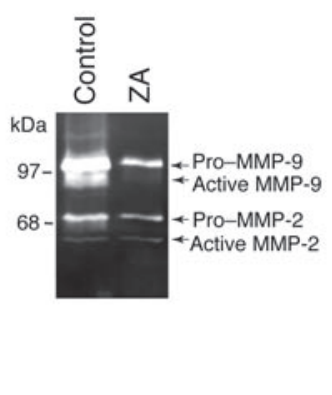

6 months later and compared with $\mathrm{HPV} / \mathrm{E}_{2}$ control mice at the same age. $\mathrm{HPV} / \mathrm{E}_{2}-\mathrm{MMP}-2-$ null mice $(n=15)$ exhibited the same tumor incidence as $\mathrm{HPV} / \mathrm{E}_{2}$ control mice $(n=40)$ and had an inconsequential $16 \%$ reduction in tumor volume $(P=0.728)$ compared with controls (Figure 7, A and $\mathrm{B})$. In contrast $\mathrm{HPV} / \mathrm{E}_{2}-\mathrm{MMP}$ $9-$ null mice $(n=25)$ evidenced a $66 \%$ reduction in tumor incidence and a $76 \%$ reduction in tumor volume $(P<0.001)$ (Figure $7, \mathrm{~A}$ and B). Immunostaining with Meca-32 $\mathrm{Ab}$ revealed reduced vessel density in $\mathrm{HPV} / \mathrm{E}_{2}$-MMP-9-/- mice, whereas no difference in vascularity was observed in HPV/E 2 -MMP-2/- mice, compared with controls (data not shown). Therefore, genetic ablation of MMP-9 demonstrated that MMP-9 activity is functionally significant for tumor progression in the cervix. Moreover, the phenotypic effects of the gene $\mathrm{KO}$ were remarkably similar to the responses seen in the ZA trails, consistent with the hypothesis that the actions of ZA involve MMP-9 as a principal target.

ZA impairs VEGF mobilization to its receptor by $M M P-9$. VEGF-A associates with its principal signaling receptor VEGF-R2 in the highly angiogenic lesions of the cervical carcinogenesis pathway (Figure 1C). Motivated by our previous observation that MMP-9 could mobilize VEGF and increase its association with VEGF-R2 (13), we sought to determine whether MMP-9 was also serving such a function in cervical neoplasias, and, if so, was ZA limiting VEGF mobilization? We analyzed the association of VEGF with VEGF-R2 in CIN-3 lesions and in tumors in ZA-treated $\mathrm{HPV} / \mathrm{E}_{2}$ mice, in MMP-2 and MMP-9 gene KO HPV/ $\mathrm{E}_{2}$ mice, and in control $\mathrm{HPV} / \mathrm{E}_{2}$ mice. As expected from unaltered tumor incidence and volume, VEGF ligand-receptor complexes were readily detected in the vasculature of MMP-2-deficient tumors (Figure 7, C and E) and CIN-3 lesions (data not shown), similar to vessels of control mice (Figure 7, C and D). In contrast, the vessels in tumors or CIN-3 lesions of MMP-9-null mice showed little or no VEGF/VEGF-R2 complex on ECs (Figure 7, C and F; the data for CIN-3 lesions are analogous but not shown). Notably, the immunostaining of vessels with GVM39 Ab in ZA-treated mice (in both prevention and regression trials) revealed a similarly reduced association of VEGF with VEGF-R2 in tumors (Figure 7, C and G) and in CIN-3 lesions (data not shown). A comparable reduction of GVM39 immunostaining (74\% reduction compared with controls) was also observed in BB94-treated cervical carcinomas (see Supplemental Figure 4, A-C) and CIN-3 lesions (data not shown), supporting the interpretation that $\mathrm{ZA}$ is inhibiting angiogenesis by interfering with MMP-9-mediated mobilization of VEGF. 
A

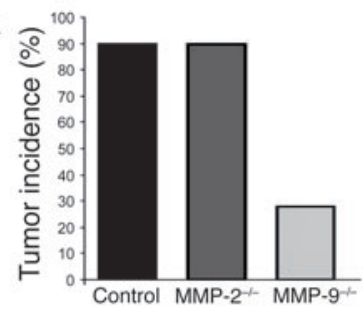

C
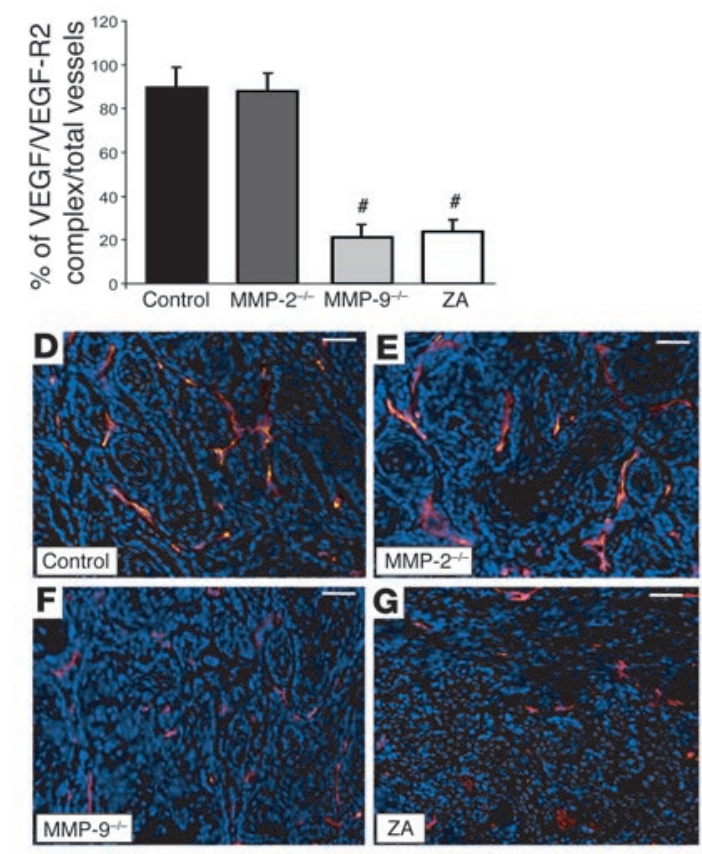

\section{Discussion}

This study has presented novel applications for an aminobisphosphonate, ZA, currently used clinically to ameliorate bone metastasis. The strategy forthcoming involves treatment of either CIN or established cervical cancer so as to elicit stasis or regression rather than progression and growth. We demonstrate that ZA inhibits angiogenesis in premalignant lesions (CIN-3) and in cervical tumors by targeting both macrophage expression and proteolytic activity of an MMP, MMP-9. Therapeutic regimes using $\mathrm{ZA}$ impaired progression of premalignant lesions to invasive carcinoma and antagonized the growth of preexisting cervical tumors. These results are likely to be relevant to human cervical carcinogenesis. MMP-9 has been shown to be upregulated in $\mathrm{CIN}-3$ lesions and cervical cancers in humans (refs. 7, 8, 36 and our unpublished observations), and elevated expression and activity correlate with poor prognosis for subsequent metastasis and tumor recurrence (37). Notably, MMP-9 and the related MMP-2 have been detected in both epithelial and stromal compartments in human cervical lesions $(8,36)$. We found MMP-9 expression exclusively in the stroma in the mouse model in infiltrating macrophages. MMP-9 is similarly expressed by macrophages (and other inflammatory cells), infiltrating both stroma and epithelia of human $\mathrm{CIN}-2 / 3$ and cervical cancers (E. Giraudo and D. Hanahan, unpublished data); the reasons that MMP-9-expressing macrophages do not infiltrate the neoplastic epithelial compartments in the mouse model are presently unclear. We performed a genetic test to assess the importance of this and a related protease by analyzing the cervical cancer phe-

\section{Figure 7}

ZA inhibits the formation of VEGF/VEGF-R2 complexes on the neoplastic vasculature, phenocopying a MMP-9 gene KO. (A) Decrease in tumor incidence in 7-month-old HPV/E $\mathrm{E}_{2}-\mathrm{MMP}^{-9-1-}$ (66\% reduction; $n=25)$ compared with HPV/E ${ }_{2}-\mathrm{MMP}^{-2^{--}}(n=15)$ and control mice $(n=40)$. (B) Reduction of tumor volume in 7-month-old $\mathrm{HPV} / \mathrm{E}_{2}-\mathrm{MMP}^{-9^{-/-}}(76 \%$ reduction; $n=25)$ compared with $\mathrm{HPV} / \mathrm{E}_{2^{-}}$ $\mathrm{MMP}^{-2^{--}}$and control mice. Tumor volume and histological scores were determined as described in Methods. (C-G) VEGF/VEGF-R2 complex was detected by immunohistochemistry using GVM39 Ab (green), while the ECs were visualized with the anti-Meca-32 Ab (red). Significant reduction in the VEGF/VEGF-R2 complex was observed on vessels in $\mathrm{HPV} / \mathrm{E}_{2}-\mathrm{MMP}-9^{-/-}$(77\% reduction compared with controls; $n=15)$ and ZA-treated $(73 \%$ reduction compared with controls; $n=8)$ mice compared with HPV/E ${ }_{2}-\mathrm{MMP}^{-2^{--}}(n=10)$ and controls $(n=20)(C)$. Representative analyses of tumors are shown in D-G. Arrows indicate the VEGF/VEGF-R2 complex associated to the vessels. Scale bar: $25 \mu \mathrm{m}$. Values are mean \pm SEM. ${ }^{\#} P<0.001$. $P$ values were calculated using the Wilcoxon test.

notype in $\mathrm{HPV} / \mathrm{E}_{2}$ mice that carried a gene $\mathrm{KO}$ of either MMP-9 or MMP-2. The HPV/E 2 -MMP-9-null mice had a markedly lower tumor incidence and a statistically significant reduction of tumor volume as well as reduced vascularity compared with control $\mathrm{HPV} / \mathrm{E}_{2}$ mice, whereas HPV/E 2 -MMP-2-null mice were largely unaffected, revealing MMP-9 as the key gelatinolytic MMP regulating cervical cancer progression in this mouse model.

These results are consistent with previous data showing in the related K14-HPV16 skin model that MMP-9 but not MMP-2 activation coincided with the angiogenic switch in premalignant lesions (18) and that MMP-9, supplied by bone marrow-derived cells, was sufficient for skin carcinogenesis in mice otherwise deficient in MMP-9 (17). Furthermore, MMP-9 but not MMP-2 has been implicated in triggering the angiogenic switch during pancreatic neuroendocrine carcinogenesis in RIP1-Tag2 transgenic mice, in part by increasing the bioavailability of VEGF-A to its receptor (13). Thus, although MMP-9 and MMP-2 have historically been considered very similar in substrate specificity, there is increasing evidence that they have distinctive targets as well as different patterns of zymogen activation. It is notable that the active form of MMP-2 was not increased in abundance, in marked contrast to the significant increase of MMP-9 activity during cervical carcinogenesis. Consistent with this difference between MMP-2 and MMP-9, RT-PCR analysis showed increased expression only of MMP-9 transcripts during carcinogenesis, whereas levels of MMP-2 and other MMPs variously implicated in malignant phenotypes, such as MMP-3, MMP-11, MMP-12, MMP-14, were unchanged, while others, MMP-8, MMP-10 and MMP-13, were not detectably expressed. Notably, the effects of genetically ablating MMP-9 were similar to those resulting from ZA therapy, consistent with the proposition that ZA's targeting of MMP-9 is indeed a key part of its biological activity in this context. Taken together, analyses of this mouse model and the cognate human cancer predict that MMP-9 functionally contributes to human cervical carcinogenesis resulting from an infiltration of neoplastic lesions by MMP-9-expressing macrophages.

$Z A$ is an unconventional metalloprotease inhibitor with multiple modes of action. Our results complement and extend a series of recent publications indicating that ZA and other BPs do more than just inhibit osteoclast functions in the bone. Several reports have documented antiangiogenic activity of ZA in cell culture and in vivo 
angiogenesis bioassays $(26,27)$ or showed that other BPs antagonize both angiogenesis and tumor growth in traditional subcutaneous xenotransplant tumor models $(28,29)$. ZA and other N-BPs also have specific effects on intracellular signaling pathways. For example, N-BPs have been shown to interfere with RAS signaling in cultured tumor cells, apparently by inhibiting the mevalonate biosynthetic pathway, which limits protein prenylation of Ras and other small GTP-binding molecules and thereby disrupts cellular functions $(38,39)$, including expression of VEGF (28). Similar intracellular effects are evident in macrophages and osteoclasts in vitro following treatment with BPs $(33,40)$. Interestingly, distinct BPs may differentially affect macrophages in different contexts: a recent study of macrophages isolated from human peripheral blood treated with two BPs, clodronate and pamidronate, revealed a complex modulation of MMP-9 expression and secretion (41), but not the suppression we observe in vivo with ZA. It is possible, therefore, that ZA has differential capability to repress MMP-9 gene expression and activity and/or that macrophages infiltrating tissues such as the cervix may respond differentially to BPs than peripheral blood monocytes; these possibilities deserve future investigation. In light of our results, it is reasonable to postulate that ZA is suppressing signaling pathways regulating MMP-9 biosynthesis and secretion by macrophages infiltrating the cervix, since we see only minimal reductions in the abundance of macrophages associated with CIN-3 and cervical cancers despite an almost complete loss of MMP-9 immunoreactivity (by IHC) and protease activity (by gelatin assay and zymography). We infer that the susceptibility of macrophages to ZA reflects their lineage relationship to osteoclasts $(42,43)$, which selectively take up BPs $(32,44)$. The biochemical mechanisms by which ZA inhibits MMP-9 gelatinolytic activity in this tissue context remains to be defined, as does the molecular basis by which it suppresses biosynthesis in macrophages. MMP-9 activity is known to be regulated at many levels (45), including gene transcription, secretion of the latent pro form, activation of the latent form by proteolytic cleavage (45-47), and enzymatic efficien$c y$ in the face of endogenous protease inhibitors. We suspect that ZA is interfering both with conversion of latent to active MMP-9 and with enzyme function, the latter due to ZA's recognized ability to chelate zinc (30), a necessary cofactor for MMP-9 activity. Finally, it is possible that ZA is affecting the interaction of MMP-9 with tissue inhibitors of metalloproteases, now known to upregulate and downregulate MMP activity through complex interactions (48).

Seeking to investigate the mechanistic basis for the observed histological responses in the neoplastic lesions, we assessed the frequency of apoptosis and found substantially increased frequencies of apoptosis in both transformed cervical epithelia and ECs, much as we and others have seen in the course of analyzing the effects on MMP-9 gene KOs and angiogenesis inhibitor therapies in other mouse models of cancer $(13,17,23)$. The data are consistent with the hypothesis that the antineoplastic responses are due to impaired angiogenesis and reduced vascularity, which results in large part from the targeting of MMP-9. Consistent with this hypothesis, we found that MMP-9-deficient $\mathrm{HPV} / \mathrm{E}_{2}$ and $\mathrm{ZA}$-treated $\mathrm{HPV} / \mathrm{E}_{2}$ mice have similarly reduced association of VEGF with VEGF-R2 in both CIN-3 lesions and cervical carcinomas. It is conceivable that $\mathrm{ZA}$ is, in addition, directly affecting the angiogenic ECs, as has been reported for cultured ECs $(26,49)$. In regard to ZA's ability to inhibit MMP-9, it is pertinent to note that MMP-9 is involved in mobilization of circulating endothelial progenitors cells (CEPs) and hematopoietic stem cells (HSCs) from the bone marrow $(50,51)$. While $\mathrm{ZA}$ has no effect on the abundance of infiltrating macrophages in cervical neoplasias, it is possible that mobilization of CEPs and HSCs is affected, which warrants future consideration.

In regard to other potential targets of $\mathrm{ZA}$ action, we cannot exclude the possibility that ZA also has a direct, proapoptotic effect on the neoplastic epithelia, and, indeed, there are reports of such activity against tumor cells in vitro $(38,52)$ and in vivo $(39,53)$. Our data show that ZA is not a traditional antimitotic chemotherapeutic drug in the cervix in that it does not reduce the proliferation rates of transformed cervical epithelial cells in CIN-3 lesions or cervical tumors. Nevertheless, we leave open the possibility that ZA is affecting the HPV16-expressing keratinocytes both indirectly (by inhibiting both MMP-9 activation and expression by macrophages and thereby impairing angiogenesis), as well as directly. Finally, we cannot rule out the possibility that MMP-9 is also antagonizing angiogenesis by generating an antiangiogenic fragment of the collagen IV $\alpha_{3}$ chain, called tumstatin, as Hamano et al. have recently shown (54); our data indicate that in balance MMP-9 is pro-angiogenic in the cervix, as shown both by ZA treatment and in HPV/E 2 -MMP-9-null mice. The cervical carcinomas are analyzed at defined end points (6-7 months of age), when the tumors are relatively small compared with the large tumors where Hamano et al. observed their effects. MMP-9 (and its inhibition by ZA) could have more complex effects in the context of large tumor burden, particularly if tumstatin mobilization by MMP-9 proves to be a factor in cervical cancer pathways. Notably, in another mouse model, that of pancreatic islet carcinoma, several classical MMP inhibitors have been found to be efficacious against early-stage but not late-stage tumors when used as monotherapy (perhaps because of the "tumstatin effect"); by contrast, a combinatorial regimen involving an MMPI along with "metronomic chemotherapy" (55) produced regression and survival benefit against large end-stage pancreatic tumors (56). This experience leads us to further suggest that ZA should also be considered for inclusion in combinatorial regimens when targeting large tumors and/or late-stage disease to circumvent a potential tumstatin effect.

We have analyzed the angiogenic phenotype and assessed a novel antiangiogenic therapy in a mouse model of cervical carcinogenesis that is driven by the etiologic viral oncogenes, faithfully recapitulating the lesional stages of the human disease. Having identified MMP-9 and its macrophage source as functional contributors to angiogenesis and tumor progression, we evaluated a targeted therapy involving a clinically approved drug, the amino-bisphosphonate $Z A$, which demonstrated promising efficacy against both premalignant lesions and cancers of the cervix. In the course of a PT, ZA inhibited angiogenesis and limited the progression of premalignant precursors to invasive carcinomas of the cervix, suggesting that ZA (or better yet an orally bioavailable derivative) might be considered for adjuvant treatment following excision of CIN-3 lesions for women at high risk for recurrence and progression. We have also shown that ZA is able to interfere with the growth of established cervical carcinomas, producing partial responses as a monotherapy. Perhaps ZA could be added to the standard of care for cervical cancer (57) to assess its potential for improving efficacy by inhibiting angiogenesis. Furthermore, given that we have identified molecular and cellular targets for ZA, thereby increasing the body of evidence $(20,58,59)$ that MMP-9 and tumor-enhancing macrophages are functionally important for angiogenesis and carcinogenesis in a variety of organs, it may be reasonable to consider incorporating ZA 
into conventional therapeutic regimens for additional tumor types and, indeed, other diseases with an angiogenic component where these biomarkers are present. In that regard, it may be pertinent to consider more frequent dosing than the current monthly schedule, which was based on ameliorating pain associated with bone metastasis and not on dose-limiting toxicity or clinical antineoplastic efficacy; instead, perhaps suppression of MMP-9 expression in tissue-infiltrating macrophages (or imaging of its protease activity in the target tissue) may be an appropriate biomarker for optimizing an efficacious antiangiogenic dosing regimen.

\section{Methods}

Transgenic mice breeding and $E_{2}$ treatment. The generation of K14-HPV16 transgenic mice (9) and $E_{2}$ treatment for cervical carcinogenesis has been previously reported $(11,12)$. Briefly, 1-month-old virgin female transgenic (heterozygous K14-HPV16 transgenic mice, line 1203\#1; see ref. 10) and nontransgenic $(\mathrm{FVB} / \mathrm{n})$ mice were anesthetized with isoflurane, and continuous-release pellets that deliver $\mathrm{E}_{2}$ at doses of $0.05 \mathrm{mg}$ over 60 days (Innovative Research of America Inc.) were implanted subcutaneously in the dorsal back skin. Subsequent pellets were implanted at 3 and 5 months of age for a total of 6 months of hormone treatment. K14-HPV16 mice were maintained in the FVB/n background (FVB/n; The Jackson Laboratory). Mice were maintained in accordance with the University of California, San Francisco institutional guidelines governing the care of laboratory mice.

MMP-9-and MMP-2-null transgenic mice. MMP-2 and MMP-9 homozygous null animals are viable and fertile, as has been reported $(34,35)$, and the absence of MMP-9 has been shown to impair skin carcinogenesis in K14HPV16 MMP-9-/- mice (17). Both MMP- $2^{+/-}$and MMP- $9^{+/-}$mice (kindly provided by Lisa Coussens, University of California San Francisco, San Francisco, California, USA) have been previously backcrossed into FVB/n to N5 and then intercrossed to produce MMP-null HPV16 animals; the $\mathrm{FVB} / \mathrm{n}$ background is permissive for squamous carcinogenesis induced by the K14-HPV16 transgene both in skin and cervix $(10,11)(\mathrm{M}$. Inoue and D. Hanahan, unpublished observations). The MMP-null HPV16 mice were treated with $\mathrm{E}_{2}$ as described above, and tumor incidence, growth, and angiogenesis were analyzed after 6 months of hormone treatment and compared to $\mathrm{HPV} / \mathrm{E}_{2}$ control mice at the same age. Histological grading and tumor volume calculation was determined as described below.

Tissue preparation and histology. Tissues were prepared as previously described for frozen and paraffin embedding $(11,13)$. Nontransgenic and transgenic mice were anesthetized with $2.5 \%$ Avertin and their hearts perfused with ice-cold paraformaldehyde (PFA) $3.75 \%$ (wt/vol) or 10\% zincbuffered formalin. The vagina, cervix, and both uterine horns were removed and postfixed in $3.75 \%$ PFA or $10 \%$ zinc-buffered formalin overnight. Ear skin was also harvested as a positive control (11). For paraffin sections, the fixed tissues were dehydrated through graded alcohols and xylene and embedded in paraffin. Five-micrometer- and $10-\mu \mathrm{m}$-thick paraffin sections were serially sectioned using a Leica 2135 microtome, deparaffinized, and rehydrated through an alcohol series, and then stained with H\&E at intervals of $100 \mu \mathrm{m}$ for histopathology and IHC. For frozen sections, the reproductive tract was embedded with or without fixation in OCT (Tissue-Tek; Sakura Finetek U.S.A. Inc.) and frozen on dry ice. Five-micrometer- and $10-\mu \mathrm{m}$-thick frozen sections were cut using a Leica CM1900 cryostat. Sections were air dried, fixed in acetone, and subjected to $\mathrm{H} \& \mathrm{E}$ staining. The characterization of neoplastic stages based on $\mathrm{H} \& \mathrm{E}$ staining have been previously reported and was done blindly (60). Tumor volume was determined using the following formula $(V=2 / 3 \times A \times Z)$, where $A$ is the cross-sectional area of the tumor determined using a Zeiss Axioskop 2 Plus equipped with a Hamamatsu-Orca digital camera and imaged using Improvision OpenLab software and $Z$ is the depth of tumor calculated through serial sections.
Drug and treatment of transgenic mice. The transgenic mice were treated from 3.5 to 5 months of age in the PT and from 5 to 6 months in the RT with the BP, ZA (Zometa; Novartis Pharma AG) designed chemically as (1-hydroxy2-imidazol-1-yl-phosphonoethyl) phosphonic acid monohydrate. ZA was dissolved in water and stored at $4^{\circ} \mathrm{C}$ as previously described (27) (for longterm storage aliquots were prepared and kept at $-80^{\circ} \mathrm{C}$ ). Mice were treated every day with $\mathrm{ZA}(100 \mu \mathrm{g} / \mathrm{kg})$ delivered by subcutaneous injection $(26,27)$. All control mice received subcutaneous water injection. BB-94/Batimastat (British Biotech Pharmaceuticals Ltd.) was homogenized in $0.02 \%$ Triton and PBS, $\mathrm{pH} 7.0$, and administered as an emulsion. Mice were treated every day for 3 months (between 4 and 7 months of age) with $25 \mathrm{mg} / \mathrm{kg}$ delivered by intraperitoneal injection (13). The animals were monitored every day during the treatment to assess side effects and were euthanized after their respective treatment period.

IHC analysis. Before use, frozen sections were air dried and acetone fixed; paraffin sections were deparaffinized and subjected to graded rehydration as previously reported (18). MMP-9 staining was carried out on paraffin sections pretreated with a 1:10 dilution of proteinase $\mathrm{K}$ for 5 minutes (DAKO Corp.). After incubation with DAKO protein block serum-free (DAKO Corp.) and a blocking solution containing PBS $(\mathrm{pH}$ 7.4) and $0.5 \%$ blocking reagent (NEN Life Science Products), the sections were stained with a 1:1,000 dilution of a rabbit anti-mouse MMP-9 Ab (61) (kindly provided by Zena Werb, University of California San Francisco, San Francisco, California, USA) in $0.5 \times$ blocking solution overnight at $4{ }^{\circ} \mathrm{C}$. After incubation with a biotinylated secondary $\mathrm{Ab}$ (goat anti-rabbit IgG; 1:200) for 30 minutes at ambient temperature, antigens were revealed with 3,3-diaminobenzidine (Sigma-Aldrich) according to the manufacturer's instructions. Sections were counterstained in $1 \%$ methyl green and visualized with Nomarski optics $(13,17)$. All images were digitally captured on a Nikon Microphot-FX microscope equipped with a DC-330 CCD color camera (DAGE-MTI of Michigan City Inc.) and imaged using Improvision OpenLab software. Frozen sections were used for immunofluorescence staining. Dilution used was 1:1,000 for rabbit anti-mouse MMP-9 and 1:200 for rat anti-mouse CD68 (MCA1957; Serotec Ltd.), rat anti-mouse F4/80 (MCAP497; Serotec Ltd.), rat anti-mouse CD8a clone 53-6.7 (BD Biosciences - Pharmingen), rat anti-mouse CD4 (H129.19; BD Biosciences - Pharmingen), rat anti-mouse CD45R/ B220 (RA3-6B2; BD Biosciences - Pharmingen), rat anti-mouse 7/4 neutrophil antigen (Cedarlane Laboratories Ltd.), rat anti-Meca32 (BD Biosciences - Pharmingen), rat anti-CD31 (MEC 13.3; BD Biosciences - Pharmingen), and 1:40 for mouse mAb GVM39 (kindly provided by Rolf Brekken, UT Southwestern Medical Center, Dallas, Texas, USA) (15) in blocking solution as described above. Dilution used for rabbit antimouse caspase-3 (Asp175; Cell Signaling Technology Inc.) was 1:100 in $5 \%$ goat serum, $1 \%$ BSA. The sections were pretreated with protein block serum-free and blocking solution as described above. The sections for caspase- 3 staining were preblocked with $5 \%$ goat serum, $1 \%$ BSA. Incubation with primary $\mathrm{Ab}$ was overnight at $4{ }^{\circ} \mathrm{C}$ followed by incubation with a cyanine3-conjugated (Cy3-conjugated) donkey anti-rabbit IgG (Jackson ImmunoResearch Laboratories Inc.) secondary Ab at 1:200 and Cy2conjugated donkey anti-rat IgG (Jackson ImmunoResearch Laboratories Inc.) for 1 hour at room temperature. To visualize blood vessels mice were first anesthetized and injected intravenously with $0.05 \mathrm{mg}$ FITClabeled tomato lectin (Lycopersicon esculentum; Vector Laboratories), and then the heart was perfused with PBS followed by $3.75 \%$ PFA. Cervices were frozen in OCT medium and sectioned at 10 and $35 \mu \mathrm{m}$. Sections were mounted in VectaShield containing DAPI (Vector Laboratories), and images were digitally captured on a Carl Zeiss Microimaging Inc. Axioskop 2 Plus, equipped with a Hamamatsu-Orca digital camera, and imaged using Improvision OpenLab software. Mast cells were visualized 
by chloroacetate esterase histochemistry as previously reported (18). All immunolocalization experiments were repeated five times on multiple tissue sections and included negative controls for determination of background staining, which was negligible.

Apoptosis and proliferation evaluation. Apoptotic cells were visualized by caspase-3 immunofluorescence as described above and on paraffin sections by TUNEL staining as previously reported (62). Proliferating cells were assessed by BrdU labeling as described (62).

Substrate zymography and gelatin assay. Gelatin zymography was performed as described (17) with the following modifications. Tissue samples were ground in liquid nitrogen, the powder weighed and then homogenized in lysis buffer containing protease inhibitor cocktail (1-873-580, EDTAfree; Roche Diagnostics $\mathrm{GmbH}$ ) as previously described (17). Equivalent amounts of soluble extract were analyzed by gelatin zymography. Data shown in Figure 2 are representative of results obtained following examination of tissue samples from mice representing distinct histological stages of neoplastic progression: $\mathrm{N} / \mathrm{E}_{2}(n=6), \mathrm{CIN}-1 / 2(n=8), \mathrm{CIN}-3(n=10)$, and $\operatorname{SCC}(n=10)$. Gelatin assay was performed as previously reported (63) with the following modifications. The samples from fresh-frozen tissue were homogenized in lysis buffer previously described (63), containing protease inhibitor cocktail (1-873-580, EDTA-free; Roche Diagnostics GmbH). Values represent the relative fluorescence units (RFU) per minute per microgram of tissue protein, averaged for four mice per control, $\mathrm{N} / \mathrm{E}_{2}$, and 1,10 phenanthroline (1,10 Phe) (Sigma-Aldrich) groups, and for three animals per ZA group (PT and RT). The experiments were repeated three times.

RNA isolation and RT-PCR analysis. Total cellular RNA was isolated from cervices and RT-PCR was performed as previously described (18) with the following modification. RNA was made using an RNeasy kit (QIAGEN Inc.). Samples were first denatured at $95^{\circ} \mathrm{C}$ for 3 minutes and amplified for 30 cycles $\left(95^{\circ} \mathrm{C} 1\right.$ minute, annealing temperature $\left[T_{\mathrm{a}}\right]=45$ seconds, $72^{\circ} \mathrm{C} 45$ seconds) and a final extension at $72^{\circ} \mathrm{C}$ for 5 minutes. Control reaction contained either control cDNA for specific primer or minus RT-RNA control. PCR products were run on $2 \%$ agarose gel. Specific primers were used to amplify cDNAs (kindly provided by Lisa Coussens): MMP-2 sense, 5'-TTGAGAAGGATGGCAAGTATGG, MMP-2 antisense, $5^{\prime}$-ACACCTTGCCATCGTTGC $\left(T_{\mathrm{a}}=55^{\circ} \mathrm{C}\right)$, 326 bp; MMP-3 sense, 5'-GACAATTCTGGAGGTTTGATGAGA, MMP-3 antisense, $5^{\prime}$-ACCAGCTGTTGCTCTTCAATATGTG $\left(T_{\mathrm{a}}=62^{\circ} \mathrm{C}\right), 207 \mathrm{bp}$; MMP-8 sense, $5^{\prime}$-TGACTCTGGTGATTTCTTGCTAA, MMP-8 antisense, $5^{\prime}$ GTGAAGGTCAGGGGCGATGC $\left(T_{\mathrm{a}}=62^{\circ} \mathrm{C}\right), 164 \mathrm{bp}$; MMP-9 sense $5^{\prime}$-GGCGTGTCTGGAGAT, MMP-9 antisense, $5^{\prime}$-AGG GTCCACCTTGTTCACC $\left(T_{\mathrm{a}}=55^{\circ} \mathrm{C}\right), 190 \mathrm{bp}$; MMP-10 sense, $5^{\prime}$-TTCTCCACAAGCCCAGCTAAC,
MMP-10 antisense, $5^{\prime}$-TGACTGAATCGAAGGACAAAGC $\left(T_{\mathrm{a}}=55^{\circ} \mathrm{C}\right)$, $171 \mathrm{bp}$; MMP-11 sense, $5^{\prime}$-ATCTCATTACCAACACCACTCC, MMP11 antisense, $5^{\prime}$-CTATGCCTACTTCCTTCGTGC $\left(T_{\mathrm{a}}=65^{\circ} \mathrm{C}\right), 527 \mathrm{bp}$; MMP-12 sense 5'-CTGCCTGTGGGGCTGCTCCCAT, MMP-12 antisense, $5^{\prime}$-ATCCTCACGCTTCATGTCCG $\left(T_{\mathrm{a}}=65^{\circ} \mathrm{C}\right), 329 \mathrm{bp}$; MMP13 sense, 5'-GACATTCTGGAACGTTATCC, MMP-13 antisense, 5'ACTCTCACAATGCGATTACTCC $\left(T_{\mathrm{a}}=55^{\circ} \mathrm{C}\right), 304 \mathrm{bp}$; MMP-14 sense, 5'-CTCTCTTCTGGATGCCCAATG, MMP-14 antisense, 5'-CACCTCAATGATGATCACCTC $\left(T_{\mathrm{a}}=55^{\circ} \mathrm{C}\right), 343 \mathrm{bp}$. Primers for GAPDH (sense, $5^{\prime}$-CCCACTAACATCAAATGGGG, antisense, $5^{\prime}$-ATCCACAGTCTTCTGGGTGG, $T_{\mathrm{a}}=55^{\circ} \mathrm{C}, 324 \mathrm{bp}$ ) were used as internal control.

Statistical analysis. Blood vessels' density and cell number were calculated counting the number of vessels or positively stained cells per field. At least five fields from each section were measured and an average per slide determined. The data were expressed as means plus or minus SEM and analyzed using the Wilcoxon test (InStat v1.12; GraphPad Software Inc.).

\section{Acknowledgments}

We thank C. Concengco, K. Gilliland, and E. Drori for excellent technical assistance and B. Bowes for support and encouragement. We wish to thank L. Coussens for generously providing the K14HPV16 MMP-9- and MMP-2-null mice inbred into the FVB/n background, $Z$. Werb for providing the polyclonal anti-mouse MMP-9 Ab, and R. Brekken for providing the GVM39 Ab. We gratefully acknowledge R.M. Gasa, J. Joyce, J. Hager, K. Smith-McCune, $Z$. Werb, and L. Coussens for helpful and insightful suggestions to the manuscript, J. Folkman for discussions, and K. Dehne for assistance and help with the gelatin assay. This research was supported by the William K. Bowes, Jr. Foundation and by grants from the US National Cancer Institute.

Received for publication May 6, 2004, and accepted in revised form July 6, 2004.

Address correspondence to: Douglas Hanahan, Diabetes Center, 513 Parnassus Avenue, HSW 1090, University of California, San Francisco, San Francisco, California 94143-0534, USA. Phone: (415) 476-9209; Fax: (415) 731-3612; E-mail: dh@biochem.ucsf.edu.

Masahiro Inoue's present address is: Department of Biochemistry, Osaka Medical Center for Cancer and Cardiovascular Disease, 1-3-3 Nakamichi, Higashinari-ku, Osaka, 537-8511, Japan.
1. Waggoner, S.E. 2003. Cervical cancer. Lancet. 361:2217-2225.

2. Clifford, G.M., Smith, J.S., Plummer, M., Munoz, N., and Franceschi, S. 2003. Human papillomavirus types in invasive cervical cancer worldwide: a metaanalysis. Br. J. Cancer. 88:63-73.

3. Smith-McCune, K.K., and Weidner, N. 1994. Demonstration and characterization of the angiogenic properties of cervical dysplasia. Cancer Res. 54:800-804.

4. Smith-McCune, K., Zhu, Y.H., Hanahan, D. and Arbeit, J. 1997. Cross-species comparison of angiogenesis during the premalignant stages of squamous carcinogenesis in the human cervix and K14-HPV16 transgenic mice. Cancer Res. 57:1294-1300.

5. Dobbs, S.P., Hewett, P.W., Johnson, I.R., Carmichael, J., and Murray, J.C. 1997. Angiogenesis is associated with vascular endothelial growth factor expression in cervical intraepithelial neoplasia. $\mathrm{Br}$. J. Cancer. 76:1410-1415.

6. Guidi, A.J., et al. 1995. Vascular permeability fac- tor (vascular endothelial growth factor) expression and angiogenesis in cervical neoplasia. J. Natl. Cancer Inst. 87:1237-1245.

7. Van Trappen, P.O., et al. 2002. A model for coexpression pattern analysis of genes implicated in angiogenesis and tumour cell invasion in cervical cancer. Br. J. Cancer. 87:537-544.

8. Asha Nair, S., Karunagaran, D., Nair, M.B., and Sudhakaran, P.R. 2003. Changes in matrix metalloproteinases and their endogenous inhibitors during tumor progression in the uterine cervix. J. Cancer Res. Clin. Oncol. 129:123-131.

9. Arbeit, J.M., Munger, K., Howley, P.M., and Hanahan, D. 1994. Progressive squamous epithelial neoplasia in K14-human papillomavirus type 16 transgenic mice. J. Virol. 68:4358-4368.

10. Coussens, L.M., Hanahan, D., and Arbeit, J.M. 1996. Genetic predisposition and parameters of malignant progression in K14-HPV16 transgenic mice. Am. J. Pathol. 149:1899-1917.

11. Arbeit, J.M., Howley, P.M., and Hanahan, D. 1996 Chronic estrogen-induced cervical and vaginal squamous carcinogenesis in human papillomavirus type 16 transgenic mice. Proc. Natl. Acad. Sci. U. S. A. 93:2930-2935.

12. Elson, D.A., et al. 2000. Sensitivity of the cervical transformation zone to estrogen-induced squamous carcinogenesis. Cancer Res. 60:1267-1275.

13. Bergers, G., et al. 2000. Matrix metalloproteinase9 triggers the angiogenic switch during carcinogenesis. Nat. Cell Biol. 2:737-744.

14. Bergers, G., and Benjamin, L.E. 2003. Tumorigenesis and the angiogenic switch. Nat. Rev. Cancer. 3:401-410.

15. Brekken, R.A., Huang, X., King, S.W., and Thorpe, P.E. 1998. Vascular endothelial growth factor as a marker of tumor endothelium. Cancer Res. 58:1952-1959.

16. Hiratsuka, S., et al. 2002. MMP9 induction by vascular endothelial growth factor receptor- 1 is involved in lung-specific metastasis. Cancer Cell. 2:289-300.

17. Coussens, L.M., Tinkle, C.L., Hanahan, D., and Werb, Z. 2000. MMP-9 supplied by bone marrow- 
derived cells contributes to skin carcinogenesis. Cell. 103:481-490.

18. Coussens, L.M., et al. 1999. Inflammatory mast cells up-regulate angiogenesis during squamous epithelial carcinogenesis. Genes Dev. 13:1382-1397.

19. Rodriguez-Manzaneque, J.C., et al. 2001. Thrombospondin-1 suppresses spontaneous tumor growth and inhibits activation of matrix metalloproteinase- 9 and mobilization of vascular endothelial growth factor. Proc. Natl. Acad. Sci. U. S. A 98:12485-12490.

20. Heissig, B., Hattori, K., Friedrich, M., Rafii, S., and Werb, Z. 2003. Angiogenesis: vascular remodeling of the extracellular matrix involves metalloproteinases. Curr. Opin. Hematol. 10:136-141.

21. Belotti, D., et al. 2003. Matrix metalloproteinases (MMP9 and MMP2) induce the release of vascular endothelial growth factor (VEGF) by ovarian carcinoma cells: implications for ascites formation. Cancer Res. 63:5224-5229.

22. Bergers, G., Javaherian, K., Lo, K.M., Folkman, J., and Hanahan, D. 1999. Effects of angiogenesis inhibitors on multistage carcinogenesis in mice. Science. 284:808-812.

23. Coussens, L.M., Fingleton, B., and Matrisian, L.M. 2002. Matrix metalloproteinase inhibitors and cancer: trials and tribulations. Science. 295:2387-2392.

24. Cohen, M.H., et al. 2002. U.S. Food and Drug Administration drug approval summaries: imatinib mesylate, mesna tablets, and zoledronic acid. Oncologist. 7:393-400.

25. Ibrahim, A., et al. 2003. Approval summary for zoledronic acid for treatment of multiple myeloma and cancer bone metastases. Clin. Cancer Res. 9:2394-2399.

26. Wood, J., et al. 2002. Novel antiangiogenic effects of the bisphosphonate compound zoledronic acid. J. Pharmacol. Exp. Ther. 302:1055-1061.

27. Fournier, P., et al. 2002. Bisphosphonates inhibit angiogenesis in vitro and testosterone-stimulated vascular regrowth in the ventral prostate in castrated rats. Cancer Res. 62:6538-6544.

28. Hamma-Kourbali, Y., et al. 2003. A novel non-containing-nitrogen bisphosphonate inhibits both in vitro and in vivo angiogenesis. Biochem. Biophys. Res. Commun. 310:816-823.

29. Sebbah-Louriki, M., et al. 2002. A new phenylacetate-bisphosphonate inhibits breast cancer cell growth by proapoptotic and antiangiogenic effects. Anticancer Res. 22:3925-3931.

30. Boissier, S., et al. 2000. Bisphosphonates inhibit breast and prostate carcinoma cell invasion, an early event in the formation of bone metastases. Cancer Res. 60:2949-2954.

31. Teronen, O., et al. 1999. MMP inhibition and downregulation by bisphosphonates. Ann. N. Y. Acad. Sci. 878:453-465.

32. Frith, J.C., and Rogers, M.J. 2003. Antagonistic effects of different classes of bisphosphonates in osteoclasts and macrophages in vitro. J. Bone Miner. Res. 18:204-212.

33. Luckman, S.P., et al. 1998. Nitrogen-containing bisphosphonates inhibit the mevalonate pathway and prevent post-translational prenylation of GTPbinding proteins, including Ras. J. Bone Miner. Res. 13:581-589.

34. Itoh, T., et al. 1998. Reduced angiogenesis and tumor progression in gelatinase A-deficient mice. Cancer Res. 58:1048-1051.

35. Vu, T.H., et al. 1998. MMP-9/gelatinase B is a key regulator of growth plate angiogenesis and apoptosis of hypertrophic chondrocytes. Cell. 93:411-422.

36. Davidson, B., et al. 1999. Expression of matrix metalloproteinase-9 in squamous cell carcinoma of the uterine cervix-clinicopathologic study using immunohistochemistry and mRNA in situ hybridization. Gynecol. Oncol. 72:380-386.

37. Sheu, B.C., et al. 2003. Increased expression and activation of gelatinolytic matrix metalloproteinases is associated with the progression and recurrence of human cervical cancer. Cancer Res. 63:6537-6542.

38. Oades, G.M., Senaratne, S.G., Clarke, I.A., Kirby, R.S., and Colston, K.W. 2003. Nitrogen containing bisphosphonates induce apoptosis and inhibit the mevalonate pathway, impairing Ras membrane localization in prostate cancer cells. J. Urol. 170:246-252.

39. Green, J.R. 2003. Antitumor effects of bisphosphonates. Cancer. 97:840-847.

40. Benford, H.L., McGowan, N.W., Helfrich, M.H., Nuttall, M.E., and Rogers, M.J. 2001. Visualization of bisphosphonate-induced caspase- 3 activity in apoptotic osteoclasts in vitro. Bone. 28:465-473.

41. Valleala, H., et al. 2003. Regulation of MMP-9 (gelatinase B) in activated human monocyte/ macrophages by two different types of bisphosphonates. Life Sci. 73:2413-2420.

42. Boyle, W.J., Simonet, W.S., and Lacey, D.L. 2003. Osteoclast differentiation and activation. Nature. 423:337-342.

43. Khapli, S.M., Mangashetti, L.S., Yogesha, S.D., and Wani, M.R. 2003. IL-3 acts directly on osteoclast precursors and irreversibly inhibits receptor activator of NF-kappa B ligand-induced osteoclast differentiation by diverting the cells to macrophage lineage. J. Immunol. 171:142-151.

44. Rogers, M.J., et al. 2000. Cellular and molecular mechanisms of action of bisphosphonates. Cancer. 88:2961-2978.

45. Nguyen, M., Arkell, J., and Jackson, C.J. 2001. Human endothelial gelatinases and angiogenesis. Int. J. Biochem. Cell Biol. 33:960-970.

46. Hahn-Dantona, E., et al. 1999. Activation of proMMP-9 by a plasmin/MMP-3 cascade in a tumor cell model. Regulation by tissue inhibitors of metalloproteinases. Ann. N. Y. Acad. Sci. 878:372-387.

47. Zhao, Y.G., et al. 2003. Activation of pro-gelatinase $\mathrm{B}$ by endometase/matrilysin-2 promotes invasion of human prostate cancer cells. J. Biol. Chem. 278:15056-15064.

48. Visse, R., and Nagase, H. 2003. Matrix metalloproteinases and tissue inhibitors of metalloproteinases: structure, function, and biochemistry. Circ. Res. 92:827-839.
49. Bezzi, M., Hasmim, M., Bieler, G., Dormond, O., and Ruegg, C. 2003. Zoledronate sensitizes endothelial cells to tumor necrosis factor-induced programmed cell death: evidence for the suppression of sustained activation of focal adhesion kinase and protein kinase B/Akt. J. Biol. Chem. 278:43603-43614.

50. Heissig, B., et al. 2002. Recruitment of stem and progenitor cells from the bone marrow niche requires MMP-9 mediated release of kit-ligand. Cell. 109:625-637.

51. Rabbany, S.Y., Heissig, B., Hattori, K., and Rafii, S. 2003. Molecular pathways regulating mobilization of marrow-derived stem cells for tissue revascularization. Trends Mol. Med 9:109-117.

52. Senaratne, S.G., Mansi, J.L., and Colston, K.W. 2002. The bisphosphonate zoledronic acid impairs Ras membrane [correction of impairs membrane] localisation and induces cytochrome c release in breast cancer cells. Br. J. Cancer. 86:1479-1486.

53. Corey, E., et al. 2003. Zoledronic acid exhibits inhibitory effects on osteoblastic and osteolytic metastases of prostate cancer. Clin. Cancer Res. 9:295-306.

54. Hamano, Y., et al. 2003. Physiological levels of tumstatin, a fragment of collagen IV alpha3 chain, are generated by MMP-9 proteolysis and suppress angiogenesis via alphaV beta 3 integrin. Cancer Cell. 3:589-601.

55. Hanahan, D., Bergers, G., and Bergsland, E. 2000. Less is more, regularly: metronomic dosing of cytotoxic drugs can target tumor angiogenesis in mice. J. Clin. Invest. 105:1045-1047.

56. Bergers, G., and Hanahan, D. 2002. Combining antiangiogenic agents with metronomic chemotherapy enhances efficacy against late-stage pancreatic islet carcinomas in mice. Cold Spring Harb. Symp. Quant. Biol. 67:293-300.

57. Engleman, M.A., and Small, W., Jr. 2003. Combined modality therapy in the treatment of gynecologic malignancies. Semin. Oncol. 30:80-94.

58. Pollard, J.W. 2004. Opinion: Tumour-educated macrophages promote tumour progression and metastasis. Nat. Rev. Cancer. 4:71-78.

59. Huang, S., et al. 2002. Contributions of stromal metalloproteinase- 9 to angiogenesis and growth of human ovarian carcinoma in mice. J. Natl. Cancer Inst. 94:1134-1142.

60. Riley, R.R., et al. 2003. Dissection of human papillomavirus E6 and E7 function in transgenic mouse models of cervical carcinogenesis. Cancer Res. 63:4862-4871.

61. Behrendtsen, O., Alexander, C.M., and Werb, Z. 1992. Metalloproteinases mediate extracellular matrix degradation by cells from mouse blastocyst outgrowths. Development. 114:447-456.

62. Lopez, T., and Hanahan, D. 2002. Elevated levels of IGF-1 receptor convey invasive and metastatic capability in a mouse model of pancreatic islet tumorigenesis. Cancer Cell. 1:339-353.

63. Rhee, J.S., Diaz, R., Korets, L., Hodgson, J.G., and Coussens, L.M. 2004. TIMP-1 alters susceptibility to carcinogenesis. Cancer Res. 64:952-961. 